\title{
EFECTO DEL CONTENIDO DE GRASA EN LA CONCENTRACIÓN DE POLIFENOLES Y CAPACIDAD ANTIOXIDANTE DE Theobroma cacao L. "CACAO"
}

\author{
Effect of fat content in polyphenols concentration and antioxidant capacity of Theobroma \\ cacao L."cacao"
}

Marili del Rosario Castro ${ }^{1}$, José A. Hernández ${ }^{1}$, Shaneri_Marcilla ${ }^{1}$, Javier S. Córdova ${ }^{1}$, Fitzgerald A. Solari ${ }^{3}$, Gabriela C. Chire ${ }^{2}$ ${ }^{1}$ Universidad Nacional Mayor de San Marcos. ${ }^{2}$ Facultad de Industrias Alimentarias, Universidad Nacional Agraria La Molina.

${ }^{3}$ Instituto Tecnológico de la Producción

\section{RESUMEN}

Los granos de cacao (Theobroma cacao L.) fueron sometidos a cuatro días de fermentación y siete días de secado natural. El contenido de polifenoles totales fue determinado por el método Folin-Ciocalteu. Los polifenoles más abundantes del cacao son metabolitos tipo catequinas flavonoides, antocianinas y proantocianinas. Estos son responsables de la actividad antioxidante y su concentración puede variar por factores de temperatura, fermentación, secado o efectos de la grasa que contiene. El contenido de polifenoles en el grano de cacao con y sin grasa fue 5,39 $\pm 0,26 \%$ y $6,26 \pm 0,26 \%$, respectivamente.

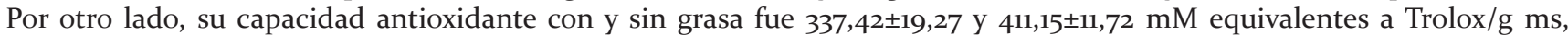
respectivamente. El contenido de polifenoles y capacidad antioxidante entre los granos grasos y desengrasados mostraron diferencias significativas $(p<0,05)$, siendo los desengrasados quienes presentaron más de ambas características, por lo que sería un proceso efectivo para el mejoramiento de su calidad nutricional dependiendo del destino.

Palabras clave: Cacao, grasa, capacidad antioxidante, polifenoles, Theobroma cacao.

\section{SUMMARY}

Cocoa beans (Theobroma cacao L.), were processed to four days of fermentation and seven days of natural drying. The content of total polyphenols was determined by Folin-Ciocalteu method. Most abundant polyphenols in cocoa are metabolits catechin flavonoid type, anthocyanins and proanthocyanins. Most of them are responsible for the antioxidant and their concentration may vary by factors such as temperature, fermentation, drying or effects due to fat content in the composition. Polyphenols content in cocoa bean with and without fat was 5,39 $\pm 0,26 \%$ and 6,26 $\pm 0,26 \%$; respectively. On the other hand, antioxidant capacity with and without fat was $337,42 \pm 19,27$ and 411,15 $\pm 11,72$ mM equivalents to TROLOX/g ms; respectively. The polyphenol content and antioxidante capacity between beans with and without fat showed significant differences $(p<0,05)$, being defatted cocoa beans the high content of polyphenols and antioxidant capacity resulting as an effective process to the improvement of nutritional quality.

Keywords: antioxidant capacity, cocoa, fat, polyphenols, Theobroma cacao.

\section{INTRODUCCIÓN}

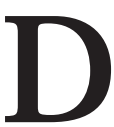
esde hace más de dos siglos, el cacao en grano se ha destacado como un rubro de gran importancia comercial en el ámbito mundial, por ser materia prima para la obtención de diversos productos de las industrias de alimentos (confitería, bebidas), cosmetológica y farmacéutica. Perú, es uno de los países productores de cacao fino y de aroma; el cacao Cutivar nativo, procedente de Piura, es uno representantes en la categoría.

El creciente interés en los últimos años por el consumo de alimentos que además de nutrir tengan un impacto favorable en la salud, ha incentivado el estudio de componentes naturales como los polifenoles presentes en plantas y frutos, los cuales han recibido especial atención debido a sus propiedades funcionales como antioxidantes, anticancerígenos, antiinflamatorios y antibacteriales ${ }^{(1)}$.

La composición química de los granos de cacao depende de varios factores entre los que se pueden citar: tipo de cacao, origen geográfico, grado de madurez, calidad de la fermentación y el secado. Sus principales constituyentes químicos son: agua, grasa, compuestos fenólicos, materia nitrogenada (proteínas y purinas), almidón y otros carbohidratos ${ }^{(2)}$.

El contenido de grasa usualmente varía del 50 al $55 \%$ en cacao fresco, estando constituida principalmente por glicéridos como el ácido oleico, palmítico y esteárico ${ }^{(3,4)}$. 
Los polifenoles de la semilla_están almacenados en células distribuidas en grupos a través de los cotiledones. La cantidad total de polifenoles solubles (porcentaje de masa seca, libre de grasa) presentes en las semillas de cacao frescas, puede variar entre 15 y $20 \%$, pero en las fermentadas esta concentración se reduce hasta un $5 \%$. Sin embargo, existen diferencias entre variedades y en algunas semillas se puede encontrar aproximadamente $2 / 3$ de la cantidad de polifenoles que hay en otras otras variedades ${ }^{(5)}$.

Debido a lo anterior, en el presente trabajo de investigación se evaluó la cantidad de polifenoles y la capacidad antioxidante del cultivar Nativo de Piura y la importancia del desengrasado_del grano con el fin de evaluar su efecto sobre el contenido de polifenoles y su capacidad antioxidante.

\section{MATERIALES Y MÉTODOS}

\section{Diseño de la investigación}

Es una investigación experimental, se desarrolló el beneficio (fermentación y secado) del Cacao, y se registró el contenido de polifenoles y capacidad antioxidante.

\section{Materia prima}

Mazorcas de cacao Cultivar Nativo ${ }^{(6)}$, procedentes del distrito de Chulucanas, provincia de Morropón, departamento de Piura, en Perú, cosechados en mayo, a 1500 a 2000 msnm.

\section{Lugar de ejecución}

Las evaluaciones se realizaron en el Laboratorio de Investigación del Instituto Tecnológico de la Producción - ITP, en Lima, Perú.

\section{Metodología}

Las mazorcas fueron abiertas para retirar el cacao en baba, iniciando el proceso de beneficio a un mismo tiempo de aguante en mazorca de cacao. La variable independiente fue el proceso de beneficio (fermentación y secado) y la variable dependiente el contenido de polifenoles y capacidad antioxidante:

Materiales: placas de metal, desecador de vidrio con agente deshidratante, tubos de ensayo, gradillas, micropipetas, espátulas, fiolas, magneto, bolsas de polipropileno, material devidrio, termómetro infrarrojo, termohigrómetro, cajas de madera tornillo de $15 \times 15$ x $15 \mathrm{~cm}$ (fermentador cuadrado ${ }^{(7)}$ con perforaciones de $0,4 \mathrm{~cm}$ de diámetro a los costados y en el fondo, para evacuar los jugos) (figura 1) y secador solar.
Equipos: estufa, incubadora con termostato, refrigeradora, molino de cuchilla (Moulinex ${ }^{\circledast}$ ), balanza analítica, balanza de precisión, centrifuga, selladora, potenciómetro, licuadora, agitador magnético, equipo de titulación, guillotina, mufla, determinador semimicro Kjeldhal, extractor Soxhlet, digestor para fibra cruda, espectrofotómetro Perkin-Elmer.

Reactivos: acetona, metanol, ácido clorhídrico, rectivo Folin-Ciocalteu, carbonato de sodio, agua desionizada, proteínas, cloruro férrico hexahidratado, tripiridil triazina (TPTZ), acetato de sodio, ácido sulfúrico y éter de petróleo, reactivo trolox, reactivo FRAP.

\section{Procedimiento}

Selección de mazorcas: se separaron frutos putrefactos, con daños físicos, dañados por insectos, germinados, etc. con el fin de obtener semillas sanas que fermenten adecuadamente y permitan obtener un producto final de alta calidad.

Quiebre: se realizó con la ayuda de un cuchillo, el cual parte a la mazorca transversalmente, con cuidado de no dañar los granos de cacao en baba.

Desgrane: se extrajo manualmente el cacao baba de 23 mazorcas para hacerlo fermentar, se separaron las raicillas y los granos pegados para evitar la formación de granos múltiples que dificulten el secado.

Fermentación: se colocaron 1,8 kg de cacao en baba en las cajas, cubriéndolas con sacos de yute para impedir la liberación de calor al exterior, finalmente fueron puestas a temperatura ambiente de $20 \pm 2{ }^{\circ} \mathrm{C}$. En el transcurso de la fermentación, las primeras 48 horas fueron sin remoción, luego de esto se realizó el volteo cada 24 horas con la finalidad de producir

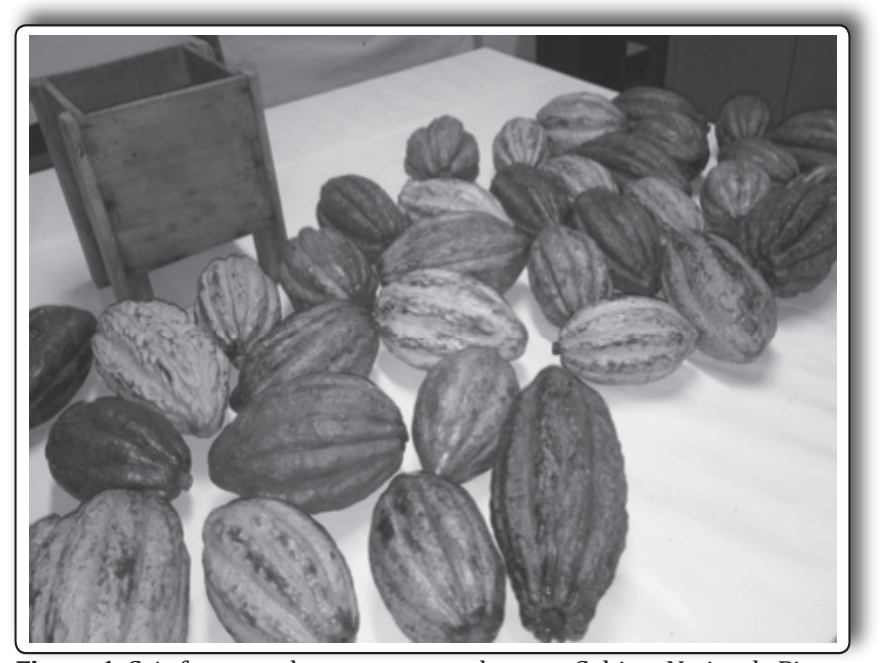

Figura 1. Caja fermentadora y mazorcas de cacao Cultivar Nativo de Piura. 
Tabla 1. Análisis proximal del grano de Cacao.

\begin{tabular}{lcc}
\multicolumn{1}{c}{ Componente } & Base humeda (\%) & Base seca (\%) \\
\hline Humedad & $55,49 \pm 0,143$ & \\
Ceniza & $1,76 \pm 0,001$ & $3,95 \pm 0,001$ \\
Proteína & $7,25 \pm 0,008$ & $16,29 \pm 0,008$ \\
Grasa & $13,67 \pm 0,004$ & $30,71 \pm 0,004$ \\
Fibra & $15,71 \pm 0,097$ & $35,30 \pm 0,097$ \\
\hline
\end{tabular}

agitar durante 1 hora. Finalmente, ambos sobrenadantes se combinaron. Se utilizó una curva estándar con ácido gálico, se tomaron los valores de absorbancia a 750 $\mathrm{ym}$, los resultados se expresaron en $\mathrm{g}$ ac. gálico / 100 g muestra. La determinación de capacidad antioxidante en los sobrenadantes se efectuó directamente

aireación y lograr que la masa alcance una temperatura homogénea ${ }^{(8,9)}$. Finalmente, transcurridos cuatro días se obtuvieron los granos fermentados.

Secado: El mismo día en la tarde, los granos de cacao fueron expuestos al medio ambiente y a la luz solar $\left(24-26^{\circ} \mathrm{C}\right)$, extendiendo la masa sobre un secador solar portátil, formando una capa de un centímetro de espesor sin granos superpuestos, variando las horas de exposición al sol y guardados en un lugar seco durante la noche ${ }^{(10)}$. El primer día fue de dos horas, luego se fue aumentando una hora diaria hasta el día siete ${ }^{(10)}$, obteniendo en total 1,2 kg de granos para determinar el grado de fermentación mediante la prueba de corte ${ }^{(11)}$.

\section{Composición química proximal del cacao fermentado}

El contenido de humedad (AOAC 931.04), cenizas (AOAC 972.15), proteína cruda (\%N x 6.25) (AOAC 970.22), grasa cruda (AOAC 920.75a) y fibra cruda (AOAC 930.20a y AOAC 962.09e) se determinaron de acuerdo a los métodos oficiales ${ }^{(12)}$. Los carbohidratos totales se calcularon por diferencia ${ }^{(13)}$ según la siguiente formula:

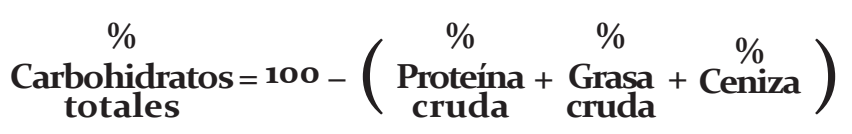

\section{Evaluación de contenido de polifenoles y capacidad antioxidante}

Los granos de cacao, previamente molidos y homogeneizados, se desengrasaron con éter de petróleo con agitación constante durante cuatro horas a temperatura ambiente y posteriormente se extrajeron sus polifenoles según el método de FolinCiocalteu (F-C) propuesto por Singleton ${ }^{(14)}$, usando $0,5 \mathrm{~g}$ de muestra que fueron tratados con $20 \mathrm{~mL}$ de metanol/agua acidificados con $\mathrm{HCl}_{2} \mathrm{~N}(50: 50 \mathrm{v} / \mathrm{v}, \mathrm{pH} 2)$, tras lo que se agitó durante una hora. Luego de centrifugar, se recogió el sobrenadante y el residuo se trató con $20 \mathrm{~mL}$ de acetona/ agua $(70: 30 \mathrm{v} / \mathrm{v})$ y se volvió a tomando alícuotas de los mismos ${ }^{(15,16)}$.

La capacidad antioxidante fue evaluada por método FRAP ${ }^{(17,19)}$, que mide la capacidad de reducción de hierro por acción de antioxidantes hidrofílicos usando la curva estándar de TROLOX (antioxidante análogo de la vitamina E). En el método, $900 \mu \mathrm{L}$ del reactivo FRAP, que contiene TPTZ, $\mathrm{FeCl}_{3}$ y tampón acetato, se mezclaron con $90 \mu \mathrm{L}$ de agua desionizada y $30 \mu \mathrm{L}$ de la muestra o el blanco (los disolventes usados para la extracción). Tras incubar a $37^{\circ} \mathrm{C}$, se tomaron los valores de absorbancia a $595 \mathrm{gm}$ a los 30 minutos, dado que a los cuatro minutos -tiempo requerido habitualmente para la formación del complejo hierro-TPTZ- la reacción aún no había terminado ${ }^{(19)}$. Los resultados se expresaron en mmoles equivalentes de Trolox / g ms.

\section{Análisis estadístico}

Todos los valores de las mediciones de polifenoles totales por F-C y de actividad antioxidante se sometieron a un tratamiento estadístico seguido del análisis de varianza ANOVA, para determinar si existen diferencias significativas entre las variables evaluadas; finalmente se usó la prueba a posteriori de Tukey, que permitió establecer las diferencias significativas entre las medias y clasificar los productos en dos grupos. Las diferencias fueron consideradas a un nivel de $p<0,05$. El análisis estadístico se realizó usando el software estadístico Minitap 16 para WINDOWS.

\section{RESULTADOS}

Los granos secos alcanzaron un grado de fermentación del $80 \%$ según la prueba de corte ${ }^{(11)}$. Los resultados de composición química proximal se observan en la tabla 1 en base húmeda y en base seca.

Tanto los granos de cacao beneficiado inicial como los posteriormente desengrasados, presentan

Tabla 2. Contenido de polifenoles y capacidad antioxidante del grano de cacao.

\begin{tabular}{lcc}
\hline \multicolumn{1}{c}{ Muestra } & $\begin{array}{c}\text { Polifenoles } \\
\text { (g ác. gálico/100g grano cacao) }\end{array}$ & $\begin{array}{c}\text { Capacidad antioxidante FRAP } \\
\text { (mM equivalente a TROLOX/g ms ) }\end{array}$ \\
\hline $\begin{array}{l}\text { Cacao beneficiado } \\
\begin{array}{l}\text { Cacao beneficiado } \\
\text { desengrasado }\end{array}\end{array}$ & $5,39 \pm 0,26^{\mathrm{a}}$ & $337,42 \pm 19,27^{\mathrm{a}}$ \\
\hline
\end{tabular}

(a) y (b) diferencia significativa $p<0,05$. 
diferencia significativa $(p<0,05)$ en el contenido de polifenoles y en la capacidad antioxidante, como se observa en la tabla 2. Debe entenderse que el término beneficiado comprende los procesos de fermentación y secado solar.

\section{DISCUSIÓN}

Los valores del contenido de polifenoles en el cacao fermentado antes y después de proceso de desengrasado fueron 5,39 y 6,26\%; respectivamente. Estos contenidos pueden estar relacionados a las temperaturas de fermentación (temperatura máxima $38-40^{\circ} \mathrm{C}$ ) y secado solar $\left(24-26^{\circ} \mathrm{C}\right)$ inicial que ayudaron a mantener los porcentajes de una manera adecuada. La literatura reporta una correlación inversa entre el contenido de polifenoles y capacidad antioxidante con las temperaturas de fermentación y secado de los granos de cacao, siendo las temperaturas de secado mayores a $40^{\circ} \mathrm{C}$ las quedisminuyen los valores alrededor de $30 \%$ del valor inicial ${ }^{(2)}$.

Zambrano ${ }^{(8)}$ encontró para el cacao criollo sin tostar, valores entre 33,97 y 44,56 mg de ácido gálico/g de grano sin tostar (equivalente a 3,397 y 4,456 g/100 g de grano sin tostar) y Rivera ${ }^{(19)}$ reporto para granos fermentados por cinco días un valor de 38,36 mg de ácido gálico/g degrano fermentado (equivalentea 3,836 $\mathrm{g} / 100 \mathrm{~g}$ de grano fermentado) y para cuatro a tres días de fermentación valores comprendidos entre 40 a $50 \mathrm{mg}$ ácido gálico/g de grano fermentado (equivalente a 4-5 $\mathrm{g}$ ácido gálico/1oog de grano fermentado), concluyendo que el tiempo de fermentación influye en el contenido de polifenoles totales. Cabe resaltar que estos valores son a partir de granos sin desengrasar por lo que serían memores al tener grasa en su composición.

Por otro lado, los valores del contenido de polifenoles del cacao beneficiado desengrasado fueron mayores en $16,1 \%$, esto debido a que la disminución de la grasa que interfiere en la cuantificación de la calidad nutricional antes mencionada.

La capacidad antioxidante muestra un patrón similar al contenido de polifenoles totales, siendo los valores obtenidos de $411,15 \mathrm{mM}$ equivalentes a TROLOX/g de materia seca, que representa el 21,9\% más que el cacao beneficiado desengrasado con 337,42 $\mathrm{mM}$ equivalentes a TROLOX/g ms de materia seca.

\section{CONCLUSIONES}

Se concluye que existen diferencias significativas $(p<0,05)$ en el contenido de polifenoles y capacidad antioxidante entre el grano de cacao (Theobroma cacao L.) en bruto y el grano de cacao beneficiado desengrasado, ambos del cultivar Nativo de Piura, siendo este proceso efectivo para incrementar el contenido de polifenoles y por tanto mejorar la capacidad antioxidante.

El desengrasado afecta la calidad nutricional, incrementado su contenido en $16,1 \%$ para polifenoles y $21,9 \%$ para su capacidad antioxidante.

\section{REFERENCIAS BIBLIOGRÁFICAS}

1. Chávez R, Ordoñez E. Polifenoles totales, antocianinas y capacidad antioxidante (DPPH y ABTS) durante el procesamiento del licor y polvo de cacao. Revista ECIPerú. 2013; 10(1): 42-50.

2. Gil J. Estabilidad y actividad antioxidante de catequinas presentes en cacaos colombianos durante los procesos de pre e industrialización. [Proyecto Tesis Doctorado]. Universidad de Antioquia. Medellín, 2012.

3. Díaz S, Pinoargote M. Análisis de las características organolépticas del chocolate a partir de cacao $\mathrm{CCN}_{51}$ tratado enzimáticamente $\mathrm{y}$ tostado a diferentes temperaturas. [Tesis de grado]. Facultad de Ingeniería Mecánica y Ciencia de la Producción. Escuela Superior Politécnica del Litoral. Guayaquil, 2012.

4. Chire G, Córdova A. Mejoramiento de chocolate amargo para taza mediante el uso de licor de cacao. Ciencia e Investigación 2005. 8 (2): 87-91.

5. VIDARIUM. Centro de investigación en nutrición salud y bienestar. Antioxidantes, chocolate y salud. Grupo Empresarial Nutresa. Medellín; 2011.

6. García LF. Catálogo de Cultivares de Cacao del Perú. $1^{\text {ra }}$ Reimpresion. Ministerio de Agricultura. Lima; 2012.

7. Portillo E, Graziani L, Betancourt E. Efecto de los tratamientos post-cosecha sobre la temperatura y el índice de fermentación en la calidad del grano del cacao criollo Porcelana (Theobroma cacao L.) en el sur del lago Maracaibo. Revista Facultad Agronomía 2005; 22(4):394-406.

8. Zambrano A, Romero C, Gómez A, Ramos G, Lacruz C, Brunetto $\mathrm{M}$ y col. Evaluación química de precursores de aroma y sabor de cacao criollo merideño durante la fermentación en dos condiciones edafoclimáticas. Agronomía Trop. 2010; 6o(2): 211-9.

9. Álvarez C, Tovar L, García H, Morillo F, Sánchez P, Girón C y col. Evaluación de la calidad comercial del grano de cacao (Theobroma cacao L.) usando dos tipos de fermentadores. Revista Científica UDO Agrícola. 2010; 10(1): $76-87$.

10. Zambrano A, Gómez A, Ramos G, Romero C, Lacruz C, Rivas E. Caracterización de parámetros físicos de calidad de almendras de cacao criollo, trinitario y forastero durante el proceso de secado. Agronomía Tropical. 2010; $60(4): 389-96$.

11. INDECOPI. NTP ISO 1114. Granos de Cacao. Prueba de corte. $3^{\text {ra }}$ ed. Lima; 2011. 
12. A.O.A.C. Official Methods of Analysis of AOAC International. Volume II. $18^{\text {th }}$ ed. Maryland: AOAC; 2005.

13. Álvarez C, Pérez E, Lares MC. Caracterización física y química de las almendras de cacao fermentadas, secas y tostadas cultivadas en la región de Cuyagua, estado de Aragua. Agronomía Tropical. 2007; 57(4): 249-56.

14. Singleton VL, Orthofer R, Lamuela-Raventós RM. Analysis of total phenols and other oxidation substrates and antioxidants by means of Folin-Ciocalteu reagent Meth Enzymol. 1999; 299: 152-78.

15. Jiménez-Escrig A, Rincón M, Pulido R, Saura-Calixto F. Guava fruit (Psidium guajava L.) as a new source of antioxidant dietary fiber. Journal of Agricultural and Food Chemistry. 2001; 49 (11): 5489-93.

16. Saura-Calixto F, Goñi I. Antioxidant capacity of the Spanish Mediterranean diet. Food Chemistry. 2006; 94(3): 442-7.

17. Benzie, I. F. F. \& Strain, J. J. The ferric reducing ability of plasma (FRAP) as a measure of "antioxidant power": the FRAP assay. Analytical Biochemistry. 1996; 239(1): 70-6.
18. Pulido R, Bravo L, Saura-Calixto F. Antioxidant activity of dietary polyphenols as determined by a modified ferric reducing / antioxidant power assay. Journal of Agriculture and Food Chemistry. 200o; 48(8): 3396-402.

19. Rivera R, Mecías F, Guzmán A, Peña M, Medina $H$, Casanova Ly col. Efecto del tipo y tiempo de fermentación en la calidad física y química del cacao ( Theobroma cacao L.) tipo nacional. Ciencia y Tecnología. 2012; 5(1): 7-12.

Manuscrito recibido el: 20/07/2015

Aceptado para su publicación el: 20/04/2016

\section{Correspondencia}

Nombre: Gabriela C. Chire Fajardo

Dirección: Av. Los Quechuas 123, Salamanca, Lima 03 - Perú.

Av. La Molina s/n, Lima 12 - Perú.

e-mail: $\quad$ gchire@lamolina.edu.pe 